\title{
Intelligent City Traffic Scheduling Optimization Based on Internet of Things Communication
}

\author{
Jingwen Jiang \\ College of Automobile and Traffic Engineering, Nanjing Forestry University, Nanjing 210037, China \\ Correspondence should be addressed to Jingwen Jiang; jiangjw@njfu.edu.cn
}

Received 13 April 2021; Revised 17 May 2021; Accepted 24 May 2021; Published 7 June 2021

Academic Editor: Wei Wang

Copyright @ 2021 Jingwen Jiang. This is an open access article distributed under the Creative Commons Attribution License, which permits unrestricted use, distribution, and reproduction in any medium, provided the original work is properly cited.

\begin{abstract}
The application of Internet of Things technology in the construction of smart city traffic system is the inevitable choice of smart city traffic system to promote the development of road traffic in the process of urban development. First of all, the traffic in the city, on the basis of the research background and significance, research on the Internet of Things, and wisdom of the construction of the urban traffic are summarized and analyzed; secondly, on the basis of requirement analysis and feasibility analysis, combined with the principles and goals of the construction of the system, the establishment of the model of urban traffic based on Internet of Things and the city's architecture based on Internet of Things intelligent urban traffic system and design are put forward. Finally, an improved ant colony algorithm is proposed in the aspect of system optimal path algorithm, and its superiority is verified. The results show that complementary networks can significantly improve the robustness of networks. These conclusions provide a theoretical basis for the construction of smart city public transport network traffic scheduling and provide model support for the operation and management of smart city public transport.
\end{abstract}

\section{Introduction}

With the continuous advancement of urbanization and the increasing urban population, the traffic pressure faced by cities is becoming more and more obvious. In order to scientifically relieve the traffic pressure and provide convenience for the emergence of urban residents, it is necessary to timely combine new technologies to promote the transformation and upgrading of the traditional traffic mode. In this development, the application of smart city traffic system is an important breakthrough. Especially with the integration of Internet of Things technology, the construction of new smart city traffic system is of great value to the alleviation of urban traffic pressure.

With wisdom of urban transport system in urban transportation planning in [1] on the intelligence development requirements also rising, under this situation, you need to in the wisdom of city traffic system actively introduce advanced Internet technology, to promote the development of the wisdom of the urban transport system intelligence and provide residents with more effective transportation service. Therefore, integrating the Internet of Things technology into the smart city traffic system is the first need to improve the intellectualization of the traffic system. As a system integrating with advanced technologies such as microelectronic sensors and wireless communication technology, the application scope of Internet of Things technology has been greatly expanded, and it also plays an important role in the construction of smart city traffic system. Through the application of Internet of Things technology, data collection and detection can be carried out more efficiently, thus providing strong technical support for the intelligent development of urban traffic. In terms of the current urban traffic management, it is generally necessary to maintain order through the consciousness of the public. The function of traffic lights and traffic signs can only provide static [2] and limited guidance, and it is difficult to realize the function of optimizing road resources. The smart city traffic system based on the Internet of Things technology can effectively collect all the road and vehicle information in the city and then dynamically calculate the best route planning to promote the orderly development of urban traffic.

Secondly, the application of Internet of Things technology is also the need to improve the level of traffic command 
and scheduling. By using the Internet of Things technology to carry out traffic command and dispatch, it can more effectively avoid the occurrence of large area of traffic congestion, so as to provide convenience for the smooth travel of residents. For example, in the process of vehicle running, the smart city traffic system of the Internet of Things can directly perceive, control, and guide road traffic by real-time processing of traffic information and using traffic signal lights, so as to achieve the function of regulating road vehicles. At the same time, with the support of RFID technology [3, 4], vehicle information can also be collected through vehicle electronic tags. This operation can realize information collection and analysis even in the high-speed operation of vehicles, thus realizing dynamic management of vehicles, ensuring accurate prediction of traffic flow, and effectively improving traffic operation efficiency.

It can be seen from the above research that the application of Internet of Things technology can promote driving safety and improve driving comfort. In the intelligent urban traffic system, improve safety and travel comfort is the most basic purpose and, with the help of the Internet of Things technology, can be achieved under the complex traffic environment, intelligent vehicle terminal based on GNSS navigation system, and the real-time data transmission on the Internet, which by the real-time display state, as far as possible to avoid congestion, avoid fault section, and improve the system efficiency.

\section{Related Work}

The EU has achieved good results in the research direction of Internet of Things technology application. In order to lead the world in the Internet of Things direction, the facility operator actively carry out the technology and application of $\mathrm{M} 2 \mathrm{M}[5,6]$.The Internet of Things application mainly includes the intelligent medical treatment, use of the sequence code product, can timely certification and drug safety, the unsafe drugs, counterfeiting, fraud has played a very good prevention, and play a blow. In addition, the application of intelligent electronic material system can provide real-time consumption information needed by consumers. Power remote monitoring system can be better in real-time and reliable grasp of the safety and use of power; in traditional industries such as logistics and retail, the application of the Internet of Things enables the timely exchange of information on various demands, meets the information needs of different groups, reduces the flow of information, and improves the efficiency of information use. The Internet of Things has been applied to People's Daily life [7, 8]. Recipe can be downloaded through mobile phone, food to be purchased can be viewed remotely through refrigerator lens, and certain activities can be automatically carried out at a certain time set on the network. With the application of various communication facilities in the Internet of Things, people can receive timely information services anytime and anywhere [9]. It is hoped to establish information standards in the new era and create a network society in the era of the Internet of Things, so as to better solve social problems such as the aging of people [10], so as to make information univer- sal and efficient popularization. At the same time, also, actively promote IoT-related industries such as strategic planning, put forward the "by building one of the world's most advanced Internet of Things from the infrastructure, the convergence of multicast communication to build future superfluid strong goal [11], from big to Internet of Things from the infrastructure, the Internet service, Internet technology, Internet of Things environment such as strength, actively carry out relevant research, looking for the Internet of Things market new business opportunities, ensuring the continuous expansion of the Internet of Things industry, and make a superb information communication technology power [12]. With the rapid development of Internet of Things, our timelines of information collection, information transmission security, and standard put forward higher request, in the process of the development of the Internet of Things is faced with many problems: lack of national standard, enterprise technology research level is weak, high cost of RFID tags, industry often lack the talent, the first technology is focused on national security question, and personal privacy is being violated not guaranteed. It can be seen that for the huge demand of IoT-related industries, how to promote the process of IoT is facing certain difficulties.

With the rapid development of science and technology and information technology, urban intelligent urban traffic has become the hot topic today; now, a lot of places are still in the stage of the urban traffic wisdom, and it is the primary stage, but the wisdom of the urban traffic is insufficient, making intelligent urban traffic must in some places to research intelligent urban traffic $[13,14]$. The smart city transportation system is guided by the framework of the national smart city transportation system to build a smart city transportation system of "high efficiency, good safety, environmental protection, and comfort." Not only improve the management level and the efficiency of the urban traffic system but also provide traffic information services, convenient, high quality, fast, economical, safe, comfortable intelligent traffic, and transportation services; improve timely, accurate, comprehensive, and sufficient information support and information-based decision support for traffic management departments and related enterprises, so as to make the system decision intelligent $[15,16]$. Many scholars are actively deploying the construction of urban smart city transportation. The research on smart city transportation is early, and the technology is advanced. Through intelligent control system, urban signal lights are controlled intelligently to reduce traffic congestion [17]. Department network intercommunication and information sharing, real-time broadcast of traffic information, traffic failure in a timely manner [18]; the use of bus priority control system to ensure the efficiency and safety of bus operation; strengthen the R\&D and application of smart city transportation, encourage the cooperation between enterprises and research institutions, etc. [19, 20]. Through the road control station, FIRD technology, advanced monitoring system, and natural traffic flow roadside system, the entering vehicles can be automatically identified, and "road congestion tax" is levied at a specific time, which effectively reduces traffic flow, reduces traffic congestion by $25 \%$, increases road traffic capacity by $80 \%$, and 
reduces vehicle exhaust emission by $8 \%-14 \%$. In addition, through the architecture of smart city transportation, good effects have been achieved in protecting environment and preventing pollution. Through sensors and road probes, traffic jams can be reported in time, and traffic jams in the next period of time can be analyzed and predicted in advance, so as to suggest smooth road driving [21,22]. Through a variety of sensors, real-time assessment of the traffic bridge can be made to obtain the number, weight, and pollution of vehicles, etc. Once the set limit value is exceeded, the traffic management department will receive an alarm, and then, find and solve the problem [23]. Through the establishment of intelligent urban traffic cloud platform founded information portal, and actively start traffic hotline, travelers can timely understand live traffic electronic bus stop, bus stations, and travel information, such as integrated traffic information query for traffic incidents, road construction, and other special circumstances; the platform can formulate and publish in time travel advice [24] and make people travel more convenient and quick. Urban transportation in the urban traffic information public platform and wisdom cloud platform to build "comprehensive three-dimensional traffic command center", to a more comprehensive, more traffic system planning and management, make "wisdom city traffic" comprehensive traffic development to upgrade, improve the intelligent level of traffic [25-27], and provide more comprehensive traffic information service for the people.

At the present stage, many cities have realized the serious problems brought by urban traffic and are actively promoting the construction of "smart city traffic," and have made certain achievements, but most of them are still in the initial stage. With the in-depth application of technologies such as the Internet of Things and cloud computing, they will surely achieve better development.

\section{Smart City Traffic System Function Based on Internet of Things}

With the rapid progress of social economy and science and technology, the city scale has been expanded to a certain extent. As a result, the traffic problem is extremely serious. At this time, in order to better solve the traffic problem, we need to do a good job in many aspects of coordination and cooperation and upgrade and optimize the urban traffic system. From the perspective of the government, when solving the problems of urban traffic development, we should not only do a good job in the introduction of new equipment but also need to increase the investment in infrastructure and traffic facilities management and further optimize the management system. The application of smart city transportation system in cities can effectively solve some practical problems and provide convenience for users. Basic functions of smart city traffic system include road condition information, service inquiry, safety guidance, and service guide [28, 29]. Smart city traffic maintains constant development in the process of continuous development. It can not only provide users with accurate traffic information but also facilitate users to query and prompt information. It has a good development prospect in the future development process. Based on the smart city traffic system, users can grasp the traffic conditions in real time and shorten the time wasted on the road. In this paper, the traffic conditions of the existing key sections are recorded and photographed, and a reasonable analysis method is adopted to classify the road levels, so as to facilitate users' travel. In the research framework, users can also query vehicle information in real time through the smart city traffic system, which can not only search vehicle information but also search vehicle information such as annual inspection, so as to provide convenience for users. Compared with relevant studies, in order to enable users to drive safely, the smart urban traffic system can remind users of traffic safety so that users can drive safely. Smart city transportation system service guide includes traffic laws, driving management business, and vehicle business. In case of problems, smart city transportation systems can provide users with legal guidance and accident counseling. On the basis of smart city traffic system, give full play to the technology of the Internet of Things and form a smart city traffic system through the in-depth application of intelligent identification, cloud computing, mobile technology, and data fusion technology. The architecture of smart city traffic system is shown in Figure 1.

Smart city transportation system can be divided into three levels from the overall architecture, namely, perception layer, network layer, and application layer.

3.1. The Perception Layer of the Internet of Things. IoT perception layer, at the end of the architecture, is the basic level in wisdom city traffic system, by using RFID, sensor networks, wireless communication and real-time positioning technology for data acquisition and collection, and timely and accurate monitoring of traffic information, it is for the whole traffic system to collect real-time traffic data for $f=$ layer for transmission and application. It consists of an M2M terminal and an infinite sensor network.

3.2. Internet of Things Network Layer. Internet network layer in the architecture of the middle layer has the effect of flow; the main principle is through the mobile network, wireless networks, Internet, satellite communication facilities such as the perception layer collected information processing to the data center, form to real information of the real reaction, through the scientific analysis of information, can provide service of traffic control decision-making.

3.3. Internet of Things Application Layer. The application layer of the Internet of Things is at the top of the architecture. Based on the various data obtained through the perception layer and the network layer, it realizes the parallel processing and optimization of massive information as well as the dynamic configuration and deployment of storage resources under the resources such as intelligent processing, cloud computing, management system, and database. Through information storage and processing system and integrated control system, information is managed, and information is presented to users in various ways for decision-making, service, and business development, including operation support platform and application system two parts. 


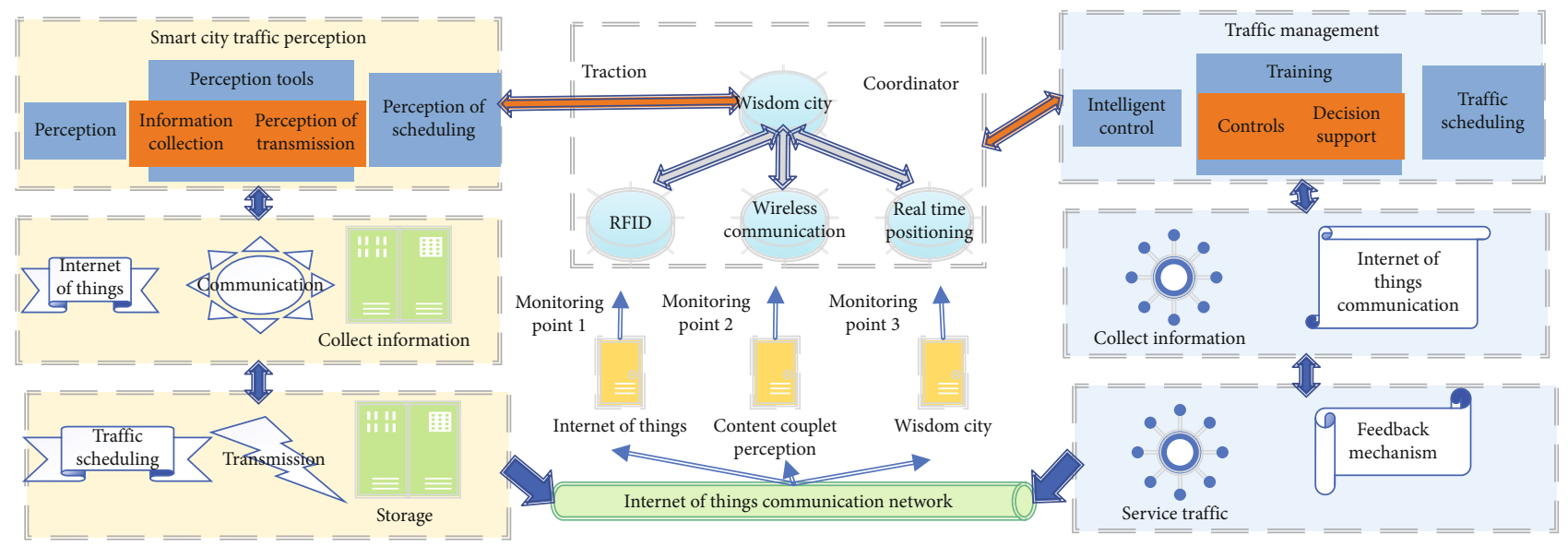

FIGURE 1: Intelligent city transportation system architecture diagram of the Internet of Things.

Wisdom urban traffic system which is built based on the Internet of Things technology in the process of general considerations should pay attention to the following several points: first, to guarantee the traffic system of dynamic, dynamic here mainly refers to during the running of the system, to be able to real-time capture different time through intelligent urban traffic system of traffic information, effective information collection, and through the data analysis response-specific traffic; second, the construction of smart city traffic system should be considered from the perspective of global, that is to say, different traffic scenes and specific application functions should be set under the same system to avoid resource waste. Third, the construction of smart city traffic system should also meet the requirements of intelligence. This construction needs to fully integrate the Internet of Things technology and promote the flexible application of Internet of Things technology in smart city traffic system, so as to master the traffic dynamics in an all-round and dynamic way. In the process of building smart city traffic system, security is the most fundamental construction point. Therefore, data security should be fully paid attention to in the construction of smart city traffic system. In the construction of the corresponding data security management system, the following four dimensions can be generally started (as shown in Table 1).

Selected by some special rule (which will be described briefly), a complete graph is formed by connecting all the possible edges with T-1 existing nodes and new nodes. Next, we will discuss degree distributions that are consistent with different rules.

(1) First, we consider how to select T-1 existing nodes. Here, we use the probability rule that is proportional to the degree $\mathrm{R}$ of each node I. Namely, the performance linear preference rule, which means that in the smart city traffic network, when a newly added station chooses to connect to a station, the station with greater performance is more likely to be selected. We can obtain the evolution equation of performance through a quasicontinuous approximation, which is very similar to the BA model. The BA model is that at the beginning, there are M0 nodes in the network, and these nodes are arbitrarily connected, as long as there is at least one link for each node, and the network evolves gradually according to growth and preference connection. Growth adds a new node with $m$ chains to the network at each step

$$
\frac{\partial T_{i}}{\partial t}=\frac{(T+1) \sum T_{i}}{(T-1) \sum T_{j}} .
$$

The performance distribution is solved as follows:

$$
p(T)=\frac{m-t \beta}{m+t \beta} T^{-(1 / \beta)}
$$

(2) We consider randomly selecting T-1 existing node rules. Following the BA model, we can write down the degree evolution equation as follows:

$$
\frac{\partial T_{i}}{\partial t}=\frac{m-t-1}{m+t}(T-1) .
$$

The urban traffic dispatching system is used to monitor commuting at important traffic crossings (such as bridges) and control the operating status of traffic lights at corresponding locations. System composition is shown in Figure 2, including bridge stress monitoring and gate access control node, traffic light control node, ZigBee-WiFi gateway, network transmission platform, acquisition service software, and web application software.

The traffic management system includes trafficintegrated information management, signal control system, police command system, and public traffic service system. Integrated traffic information management is to understand the real-time use level of urban traffic on the basis of traffic data collection and provide reasonable travel suggestions for travelers in combination with the theory of traffic planning in reality. Signal control system is through the management of traffic infrastructure, to understand the reality of 
TABLE 1: Basis of safety management system construction.

\begin{tabular}{lcc}
\hline Security dimension & Object-oriented & Take measures \\
Application & System users & $\begin{array}{r}\text { It can be controlled by access control, data encryption, protocol } \\
\text { modification, and other measures }\end{array}$ \\
System & All system data & $\begin{array}{r}\text { It is necessary to strengthen the protection of basic service software } \\
\text { security vulnerabilities, such as database and operating system } \\
\text { The data generated by the Internet of Things are encrypted and } \\
\text { transmitted through the password algorithm and then transmitted } \\
\text { through the Internet. After data sharing, the function of data fusion in } \\
\text { the intelligent system is achieved }\end{array}$ \\
Data & All application data & $\begin{array}{r}\text { Through the establishment of smart city traffic dedicated platform to } \\
\text { deploy software and store data }\end{array}$ \\
\hline
\end{tabular}
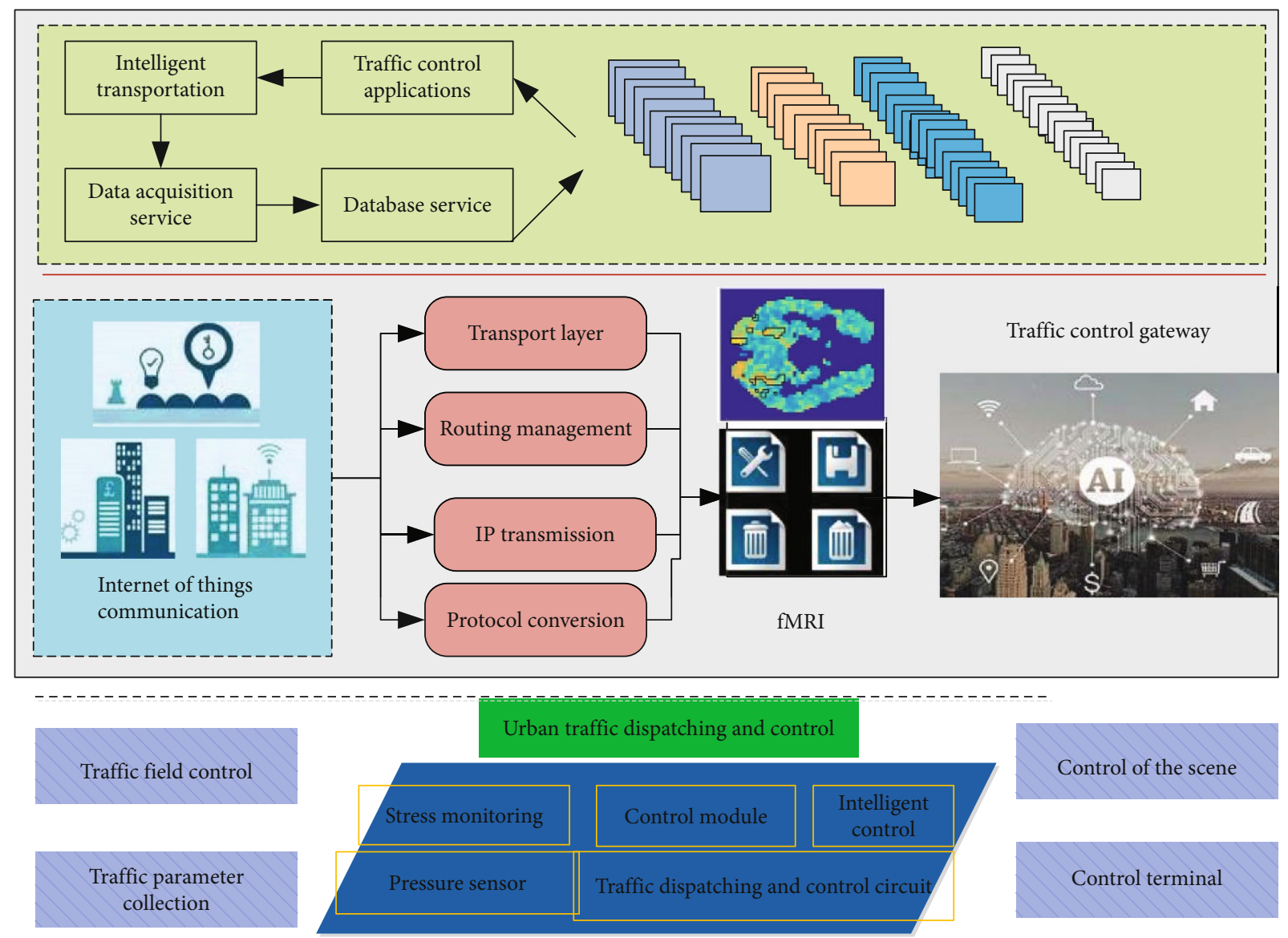

Figure 2: Block diagram of urban traffic dispatching and control system.

urban traffic flow and traffic congestion information and take a reasonable control of the signal indication, so that the traffic flow of the road will reach a reasonable level. The police command system handles emergencies in traffic, obtains road sections and traffic conditions through traffic facilities, releases information in time, and takes corresponding measures to reduce the traffic impact caused by the events. The public transport service system enables travelers to timely understand the travel route, traffic location, and other information; reasonably arrange the transportation mode; and optimize the travel activities.

\section{Research on Intelligent City Traffic Scheduling Optimization Algorithm Based on Internet of Things}

In the real traffic, the optimal path selection in the traffic network is different from the shortest path problem in theory. One-way driving on road sections, turning restrictions at intersections, as well as optimization standards for the shortest driving distance, the lowest driving cost, and the least traveling time will all have corresponding influences on the optimal path for traffic [27], which shows that people have 
different requirements for real-time path in traffic. The relevant influencing factors are mainly divided into the following three aspects.

4.1. Real-Time Traffic Flow. In real traffic, if there are more vehicles on an optimal path from the starting point to the destination, which exceeds the carrying capacity of the path, urban traffic congestion is bound to result. The optimal path planning in order to be more in line with the actual must be according to the traffic flow to quantify the degree of congestion; traffic congestion discriminant is of high maneuverability, average travel speed of the motor vehicle can root for description, and concrete can be divided into smooth, mild crowded, crowded, and severe degree of four.

4.2. Delay Time at the Intersection. In mathematics, path optimization regards each node as an abstract point with zero delay, whose existence does not affect the optimization time and does not play any role. However, in the actual traffic network, the influence of traffic lights and other conditions is very obvious, which inevitably leads to time delay. Its weight in the optimal traffic time path is relatively large, so it can be seen that the delay time generated when vehicles pass the crossing day must be considered in the path optimization algorithm.

4.3. The Influence of One-Way Traffic. Under normal circumstances, the traffic administrative department of the sections in order to optimize certain set up of the road, the intelligent drive from one end to the other side cannot reverse driving, and general path algorithm is assumed that any two nodes are exchanged; in this case, set in accordance with the route is not realistic, so you must consider road affects traffic path.

In the process of solving the optimal traffic path, the basic ant colony algorithm has some problems, such as long searching time and easy to converge to the local optimal solution. In the actual traffic, there exists the problem of steering direction. By introducing the directional guidance information and choosing the preferred traversal path, the efficiency of the algorithm can be improved. The problem of search converging to the local optimal solution is solved by the cooperative characteristic between ant colonies, and the accuracy of search is improved.

Because the basic ant colony algorithm has no directionality when solving the shortest path in the complex traffic network, the directionality has a great weight when solving the shortest path by improving the visibility parameter on the basis of the transition probability.

$$
\mu_{j k}=\frac{1-\omega_{k j} \cdot \beta_{j k}}{1+\omega_{k j} \cdot \beta_{j k}} .
$$

Because of the influence of pheromone on path selection, the pheromone can be divided into the starting point pheromone and the end point pheromone. Each ant starts at the beginning and disperses the most pheromones at the beginning and the end. As the distance goes, the amount of pheromone disperses decrease. Individual ants have only one type of pheromone, which changes when they reach their destination. After $N$ iterations, the path with the most pheromones is the shortest path. According to the collection of the starting point and the end point, the shortest path from the starting point to the end point can be found.

In order to avoid the excessive accumulation of road pheromones and prevent the search from converging to the local optimal solution, it is necessary to ensure the diversity of path choices and explore different paths to find the optimal solution, which requires the local update of pheromones. In the updating process, the pheromone concentration is updated for the road section passed by ants according to the local updating rules. The local update rule is shown in the formula.

$$
\lambda(j, k)=\frac{\lambda_{j k}-\Delta \lambda_{j k}^{t}}{2} .
$$

Theoretically, if the pheromone concentration of a certain road is too low, the probability of ants' choice will be affected, or even cannot be chosen by ants. If so, the diversity of path choices will be affected, so that the optimal solution may not be the optimal path. Therefore, a minimum standard can be set for the pheromone concentration of the road. When the pheromone concentration of the road section is lower than this standard, it is forced to restore to the minimum standard line to ensure the diversity of route selection. Local pheromone updating can effectively solve the problem of excessive accumulation of path pheromones, prevent the possibility of local convergence, and expand the search scope as much as possible, which is conducive to the selection of the optimal path.

Step 1. Initialize parameters, and the number of iterations is 0 .

Step 2. Set the maximum number of iterations, increasing by iteration $N=N+I, A, 1$.

Step 3. Set $A=A+1$.

Step 4. Ant A moves to the next node $\mathrm{k}$ according to the transition probability.

Step 5. Modify taboos and update pheromones locally.

Step 6. If node $\mathrm{k}$ is the end point, perform Step 7. Otherwise, jump to Step 4 .

Step 7. If A is greater than the total number of ants, perform Step 8; otherwise, skip to Step 3.

Step 8. Update the global pheromone.

Step 9. If the maximum number of iterations is satisfied, perform Step 10; otherwise, perform Step 2.

Step 10. Finish the search and output the optimal result. 


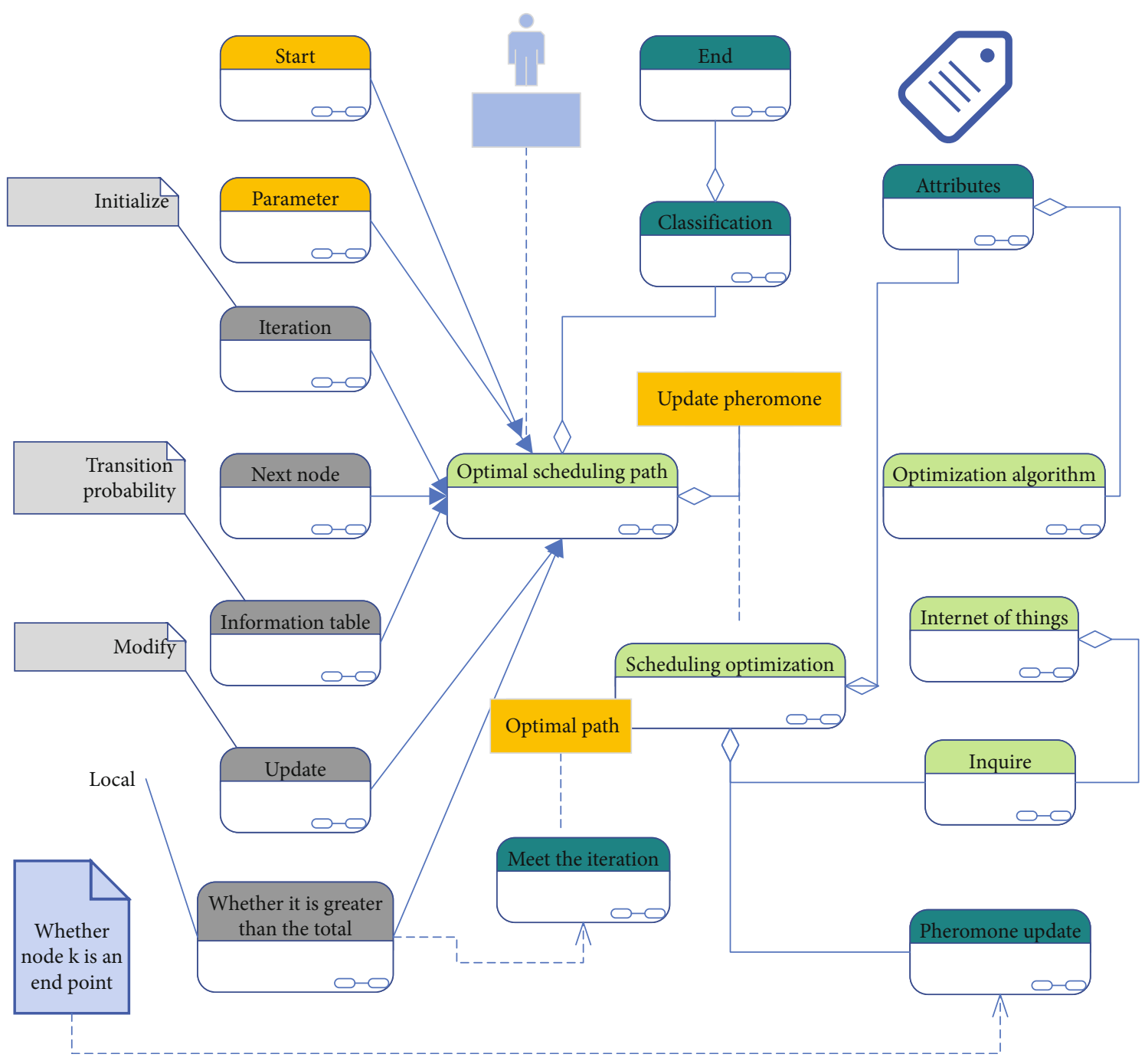

FIGURE 3: Intelligent city traffic scheduling optimization algorithm for the Internet of Things.

TABLE 2: Descriptive statistics of main variables.

\begin{tabular}{|c|c|c|c|c|c|}
\hline Variable & Clarification & Mean & Standard deviation & Minimum value & Maximum \\
\hline $\mathrm{TP}$ & \multirow{4}{*}{ First map } & 22781.48 & 74785.23 & 78.02 & 1132569.21 \\
\hline GDP & & 59636.5 & 49872.45 & 4410.186 & 506301.2 \\
\hline FDI & & 0.0033 & 0.0046 & 0 & 0.0564 \\
\hline STE & & 0.0171 & 0.0164 & 0.0003 & 0.2313 \\
\hline Ind & \multirow{4}{*}{ Second path } & 50.0139 & 11.8912 & 9.73 & 87.94 \\
\hline EDU & & 470.4439 & 409.3027 & 0 & 2409.313 \\
\hline POP & & 0.1026 & 0.0944 & 0.0023 & 1.1448 \\
\hline BUS & & 119.4722 & 113.5525 & 1.9540 & 1591.47 \\
\hline INN & \multirow{3}{*}{ Inner path } & 12.44 & 55.65 & 0.006 & 1061.27 \\
\hline EMP & & 38.75 & 84.45 & 1.13 & 923.2 \\
\hline $\mathrm{TECH}$ & & 2.313 & 1.894 & 0.077 & 12.152 \\
\hline
\end{tabular}

The improved algorithm flow is shown in Figure 3.

The changed algorithm emphasizes the directionality of the transfer, the classification of the pheromone, and local pheromone updates and enables the ants to explore new paths, so it can strengthen the basic ant colony algorithm of pheromone positive feedback, improve the difference of each section of pheromone concentration, and prevent to explore the path of reducing, so as to improve the efficiency of the algorithm. The main variable design is shown in Table 2. 


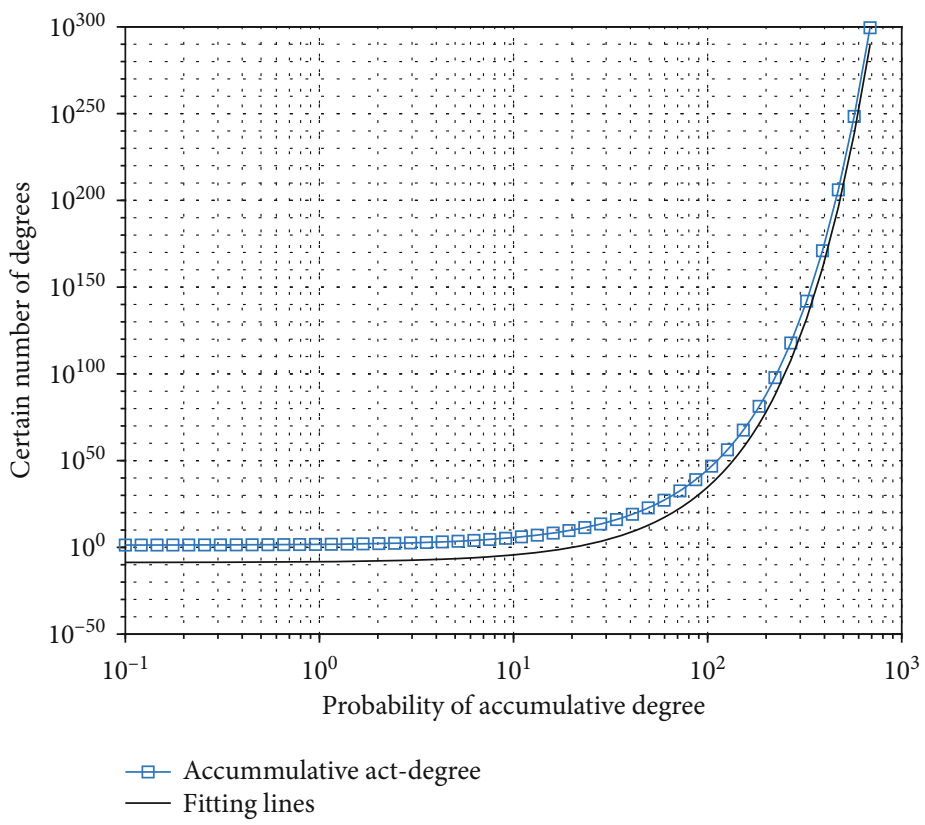

FIGURE 4: Distribution diagram of traffic scheduling accumulation degree in smart city.

TABLE 3: Network model characteristic parameter simulation data table.

\begin{tabular}{lcccc}
\hline $\begin{array}{l}\text { Network parameters and } \\
\text { characteristic quantities } \\
\text { (simulation data) }\end{array}$ & City 1 & City 2 & City 3 & City 4 \\
\hline Number of sites & 165 & 245 & 123 & 94 \\
Number of lines & 12 & 13 & 7 & 6 \\
The network diameter & 5 & 5 & 2 & 2 \\
The average degree & 21.65 & 25.49 & 21.59 & 24.58 \\
Mean path length & 2.374 & 2.523 & 2.182 & 1.696 \\
Clustering coefficient & 0.9069 & 0.9033 & 0.8102 & 0.8335 \\
\hline
\end{tabular}

\section{Results Analysis}

We has carried on an empirical analysis of a city traffic scheduling in 2017, which includes 736 city bus lines and 5487 bus site; in order to reflect the geographical topology characteristics of the web site of the bus, we adopt L space method, namely, each bus station is defined as a node, if two nodes in the same bus line when they are connected by an edge. As shown in Figure 4, we show the cumulative degree distribution of urban traffic scheduling. In Figure 4, the vertical axis represents the probability of accumulative degree, and the horizontal axis represents a certain number of degrees.

The smart city traffic scheduling network model of Internet of Things communication is used for digital simulation, and the results of digital simulation are compared with the results of empirical analysis. Table 3 shows the simulated calculation values of network characteristic statistical parameters. The simulated data are basically consistent with the empirical analysis data. This network model can better explain the characteristics of smart city traffic scheduling in the Internet of Things communication.

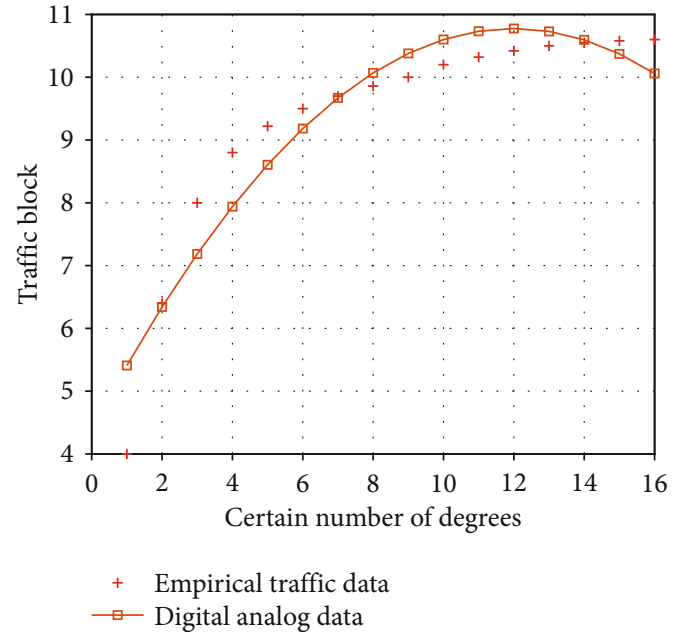

FIgURE 5: Comparison between simulation data and empirical data of urban transport network cumulative degree distribution.

The circular data curves in Figure 5, respectively, represent the simulation graph of the accumulation degree distribution with the urban transport network. It can be seen that the simulation graph is consistent with the results of the empirical analysis, showing a segmented degree distribution law.

According to the simulation results of network nodes supplemented by random attacks (Figure 6), the network evolution law is basically similar. As stated in the simulation modeling method, random attacks cover the phase transition points of system collapse after 50 configuration averaging, so it is impossible to make quantitative comparison. However, according to the evolution law of the average path length, there is an obvious rising process in the attack process, because there are fewer routes but more stations in each route. 

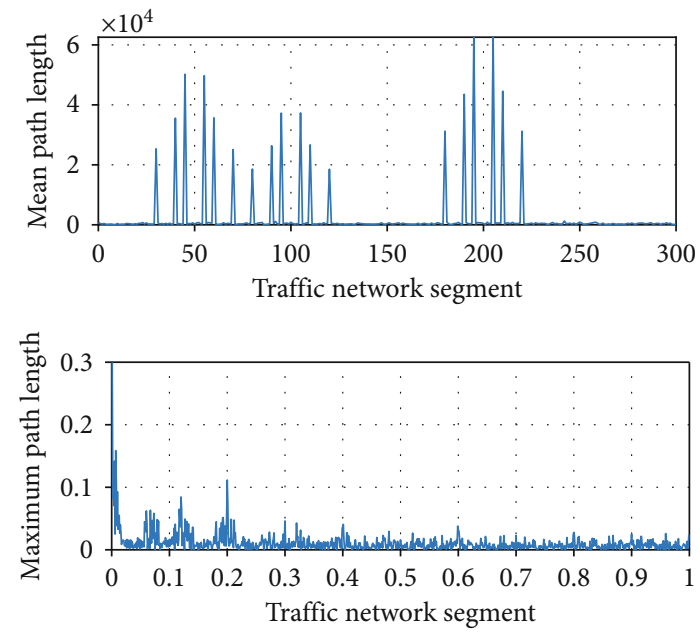

FIGURE 6: Simulation results of the robustness of random attacks complementing each other.
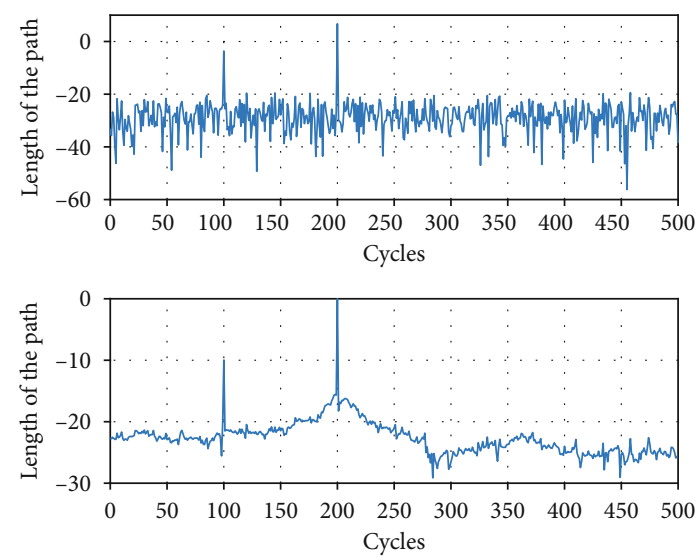

FIGURE 7: Search process of improved scheduling algorithm.

Parameter settings of smart city traffic scheduling algorithm are based on Internet of Things communication. Figure 7 shows the search process of scheduling algorithm and shows the optimal path length found by scheduling in each round of search.

\section{Conclusion}

To sum up, on the basis of demand analysis and feasibility analysis, combined with the principles and objectives of system construction, this paper establishes the urban traffic model based on the Internet of Things and puts forward the architecture and application architecture of the intelligent transportation system focusing on system integration and application. It is necessary to apply the intelligent transportation system of Internet of Things technology to improve the low efficiency of traffic information collection and the traditional intelligent system of imperfect traffic data, so as to promote the efficient development of urban traffic system. The results show that complementary networks can significantly improve the robustness of networks. These conclusions provide a theoretical basis for the construction of smart city pub- lic transport network traffic scheduling and provide model support for the operation and management of smart city public transport.

\section{Data Availability}

The data used to support the findings of this study are available from the corresponding author upon request.

\section{Conflicts of Interest}

The authors declare that they have no known competing financial interests or personal relationships that could have appeared to influence the work reported in this paper.

\section{References}

[1] X. Fei and G. Z. Tian, "Optimization of communication network fault identification based on NB-IoT," Microprocessors and Microsystems, vol. 80, pp. 103531-103545, 2021.

[2] L. Huang, X. Yuan, J. Zhang, N. Zhang, J. Li, and L. Wang, "Research on internet of things technology and its application in building smart communities," Journal of Physics: Conference Series, vol. 1550, no. 2, pp. 22029-22031, 2020.

[3] M. M. Rathore, A. Ahmad, A. Paul, and S. Rho, "Urban planning and building smart cities based on the Internet of Things using big data analytics," Computer Networks, vol. 101, pp. 6380, 2016.

[4] X. Meng, "Urban planning and building smart cities based on the Internet of Things using big data analytics," Computing Reviews, vol. 58, no. 10, pp. 608-618, 2017.

[5] W. Li, W. Xue, and S. Hou, "Analysis on bilingual public signs in the view of audience theory-a case study of city traffic public signs in Xi'an," Open Journal of Modern Linguistics, vol. 5, no. 2, pp. 181-186, 2015.

[6] Y. Zhao, J. Ma, L. Shen, and Y. Qian, "Optimizing the junction-tree-based reinforcement learning algorithm for network-wide signal coordination," Journal of Advanced Transportation, vol. 2020, Article ID 6489027, 11 pages, 2020.

[7] F. Tang, "Intelligent bionic optimization algorithm based on the growth characteristics of tree branches," International Journal of Cognitive Informatics and Natural Intelligence, vol. 15, no. 2, pp. 34-46, 2021.

[8] C. X. Mavromoustakis, G. Mastorakis, and J. M. Batalla, "Internet of things (IoT) in 5G mobile technologies signal processing techniques for energy efficiency, security, and reliability in the IoT domain," in Modeling and Optimization in Science and Technologies, pp. 419-447, Springer-Verlag, 2016.

[9] W. H. Wei, Y. N. Zhu, T. M. Xue, S. Y. Xie, C. R. Yuan, and T. Y. Wang, "Optimization of the frame and performance analysis of solar modules based on Ansys workbench and Solidworks," Key Engineering Materials, vol. 693, pp. 169$173,2016$.

[10] G. Li, S. Fang, J. Ma, and J. Cheng, "Modeling merging acceleration and deceleration behavior based on gradient-boosting decision tree," Journal of Transportation Engineering, Part A: Systems, vol. 146, no. 7, pp. 2723-2737, 2020.

[11] Y. Y. Pan, "Lagrangian relaxation for the multiple constrained robust shortest path problem," Mathematical Problems in Engineering, vol. 2019, Article ID 3987278, 13 pages, 2019. 
[12] X. Du, Z. Zhu, J. Chen, C. Qi, and X. Guo, "Route configuration method for highway passenger hubs from the perspective of transportation integration: a case study of Nanjing, China," Symmetry, vol. 12, no. 7, pp. 1194-1205, 2020.

[13] M. Jarrah, M. Jaradat, Y. Jararweh, M. Al-Ayyoub, and A. Bousselham, "A hierarchical optimization model for energy data flow in smart grid power systems," Information Systems, vol. 53, no. 12, pp. 190-200, 2015.

[14] C. Lin, Y. Bi, H. Zhao, Z. Liu, S. Jia, and J. Zhu, "DTE-SDN: a dynamic traffic engineering engine for delay-sensitive transfer," IEEE Internet of Things Journal, vol. 5, no. 6, pp. 52405253, 2018.

[15] J. Jiao, Y. Sun, S. Wu, Y. Wang, and Q. Zhang, "Network utility maximization resource allocation for NOMA in satellite-based Internet of Things," IEEE Internet of Things Journal, vol. 7, no. 4, pp. 3230-3242, 2020.

[16] S. Xu, X. Wang, G. Yang, J. Ren, and S. Wang, "Routing optimization for cloud services in SDN-based Internet of Things with TCAM capacity constraint," Journal of Communications and Networks, vol. 22, no. 2, pp. 145-158, 2020.

[17] M. A. Al Mamun, M. A. Hannan, and A. Hussain, "Theoretical model and implementation of a real time intelligent bin status monitoring system using rule based decision algorithms," Expert Systems with Applications, vol. 48, pp. 76-88, 2016.

[18] X. Tian, Y. Cheng, D. M. Shila, and A. Wolisz, "Guest editorial special issue on enabling a smart city: internet of things meets AI," IEEE Internet of Things Journal, vol. 6, no. 5, pp. 7469$7472,2019$.

[19] H. J. Feng, L. G. Chen, Z. H. Xie, and F. R. Sun, “"Volumepoint" heat conduction constructal optimization based on minimization of maximum thermal resistance with triangular element at micro and nanoscales," Journal of the Energy Institute, vol. 89, no. 2, pp. 302-312, 2016.

[20] M. Yuan, Y. Li, L. Zhang, and F. Pei, "Research on intelligent workshop resource scheduling method based on improved NSGA-II algorithm," Robotics and Computer-Integrated Manufacturing, vol. 71, no. 12, pp. 102141-102149, 2021.

[21] T. Guo and Z. Deng, "An improved EMD method based on the multi-objective optimization and its application to fault feature extraction of rolling bearing," Applied Acoustics, vol. 127, pp. 46-62, 2017.

[22] J. Yang, J. Zhou, Z. Lv, W. Wei, and H. Song, “A real-time monitoring system of industry carbon monoxide based on wireless sensor networks," Sensors, vol. 15, no. 11, pp. 29535-29546, 2015.

[23] H. Hu, B. Tang, X. Gong, W. Wei, and H. Wang, "Intelligent fault diagnosis of the high-speed train with big data based on deep neural networks," IEEE Transactions on Industrial Informatics, vol. 13, no. 4, pp. 2106-2116, 2017.

[24] W. Wei, Q. Xu, L. Wang et al., "GI/Geom/1 queue based on communication model for mesh networks," International Journal of Communication Systems, vol. 27, no. 11, pp. 30133029, 2014.

[25] F. Orujov, R. Maskeliūnas, R. Damaševičius, W. Wei, and Y. Li, "Smartphone based intelligent indoor positioning using fuzzy logic," Future Generation Computer Systems, vol. 89, pp. 335-348, 2018.

[26] W. Wei, S. Liu, W. Li, and D. Du, "Fractal intelligent privacy protection in online social network using attribute-based encryption schemes," IEEE Transactions on Computational Social Systems, vol. 5, no. 3, pp. 736-747, 2018.
[27] L. Jiang, L. Chen, W. Wang, W. Wei, Z. Lv, and H. Wang, "Advanced network representation learning for container shipping network analysis," IEEE Network, vol. 35, no. 2, pp. 182-187, 2021.

[28] N. A. Khan, N. Z. Jhanjhi, S. N. Brohi, R. S. A. Usmani, and A. Nayyar, "Smart traffic monitoring system using unmanned aerial vehicles (UAVs)," Computer Communications, vol. 157, pp. 434-443, 2020.

[29] F. Outay, H. A. Mengash, and M. Adnan, "Applications of unmanned aerial vehicle (UAV) in road safety, traffic and highway infrastructure management: recent advances and challenges," Transportation Research Part A Policy and Practice, vol. 141, pp. 116-129, 2020. 